\title{
Optimization Of Queueing Model
}

\section{Dr. Navneet Kumar Verma ${ }^{1}$, Dr. Shavej Ali Siddiqui ${ }^{2}$}

${ }_{1}^{1}$ (Associate Professor) in Mathematics Department, VIT Bhopal University, Madhya Pradesh

${ }^{2}$ Assistant Professor' in the Dept. of Mathematics, Khwaja Moinuddin Chisti Language University, Lucknow, Uttar Pradesh

Article History: Received: 11 January 2021; Accepted: 27 February 2021; Published online: 5 April 2021

\begin{abstract}
: -in thepaper, we are considering the single server queueing system have interdependent arrival of the service processes having bulk service.In this article, we consider that the customers are served $k$ at any instance except when less then $k$ are in the system \&ready to provide service at which time customers are served.
\end{abstract}

Keyword: -Interdependent queueing models, arrival process, service process, waiting line system, mean dependence.

\section{OPTIMIZATION $M / M^{[\mathrm{K}]} / 1$ QUEUEING MODEL WITH VARYING BATCH SIZE :-}

In this type of systems, the interdependence could be induced by considering the dependent structure with parameters $\lambda, \mu$ and $\in$ as marginal arrival rate, service rate and mean dependence rate respectively .

Let $P_{n}(t)$ be the probability when there are $n$ customers in system at time $t$. The difference - differential equations of above modelmay have written as,

$$
\begin{aligned}
& P_{n}^{\prime}(t)=-(\lambda+\mu-2 \in) P_{n}(t)+(\lambda-\epsilon) P_{n-1}(t)+P_{n-k}(t) ; n \geq 1 \\
& P_{0}^{\prime}(t)=-(\lambda-\epsilon) P_{0}(t)+(\mu-\epsilon) \sum_{i=1}^{k} P_{i}(t)
\end{aligned}
$$

Let us considerthat, the system achieved the steady state, therefore the transition equations of considered model ar,

$$
\begin{gathered}
-(\lambda+\mu-2 \epsilon) P_{n}+(\lambda-\epsilon) P_{n-1}+(\mu-\epsilon) P_{n-k}=0 \quad ; n \geq 1 \\
-(\lambda-\epsilon) P_{0}+(\mu-\epsilon) \sum_{i=1}^{k} P_{i}=0
\end{gathered}
$$

Applying heuristic arguments of "Gross and Harris" (1974). One can obtain the solution of mentioned equationsas,

$$
P_{n}=C r^{n} n \geq 0 \quad, \quad 0<r<1
$$

Where $r$, is the root of equations which lie in $(0,1)$ of the characteristic equation .

$\left[(\mu-\epsilon) D^{k+1}-(\lambda+\mu-2 \epsilon) D+(\lambda-\epsilon)\right] P_{n}=0$

Here Drepresents theoperator.

\section{MEASURES OF EFFECTIVENESS: -}

The probability that the system is empty is,

$$
P_{0}=(1-r)
$$

Where $r$ is as given in equation (3).

For different values of $\in \& k$, for the given values of $\lambda$ and $\mu$, we are able to compute $P_{0}$ values $\&$ are given in table (5.1). The values of $P_{0}$ for the fixed $k$, eand for varying $\lambda, \mu$ mentioned in the table (5.2).

From tables (5.1), (5.2) and equation number---(5), we observe that for fixed of $\lambda, \mu$ and $\in$, the value of $P_{0}$ increases with respect to increase in $k$. As the dependence parameter $\in$ increases the value of $P_{0}$ increases for fixed values of $\lambda, \mu$ and $k$.The value of $P_{0}$ decreases for fixed values of $\mu, k$ and $\in$ as $\lambda$ increases. As $\mu$ increases the valueof $P_{0}$ increases for fixed values of the $\mu, k$ and dependence parameter $\epsilon$. If the mean dependence rate, is zero then the value of $P_{0}$ is also same as in the $M / M^{[K]} / 1-$ model.

The average no. of customers in the system can obtained as

$$
L=\frac{r}{1-r}
$$

and mean number, of customers in the queue are

$$
L_{q}=\frac{r^{2}}{1-r}
$$


where $r$ is as given in equation (3).

The value of $L$ and $L_{q}$ has been computed and given in tables-5.3 and table-5.5 for provided values of $\lambda, \mu$ and for different values $\in$ and $k$ respectively. The values of $L$ and $L_{q}$ for fixed values of $\in$ and $k \&$ for varying $\mu$ and $\lambda$ also given in tables-5.4 and table-5.6.

By equations 6 and 7, also for the corresponding tables we observe, that as Eincrease, the values of $L$ and $L_{q}$ are decreasing and also as $k$ increases the values of $L$ and $L_{q}$ are decreasing for fixed values of other parameters. As the arrival rate increases, the values of $L$ and $L_{q}$ are increasing for fixed values of $\mu, k$ and $\in$. As $\mu$ increases the values of $L$ and $L_{q}$ are decreasing for fixed values of $\lambda, k$ and $\in$. When the dependence parameter $\epsilon=0$ then the average queue length is same as that of $M / M^{[K]} / 1$ model. When $k=1$ this is same as $M / M / 1$ interdependence model.

The variability of this model can be obtained as

$$
V=\frac{r}{(1-r)^{2}}
$$

where $r$ is as given in equation (3).

The coefficient of variation of the model is

$$
C . V=\frac{\sqrt{V}}{L} \times 100
$$

Where L \& V are provided as in equations (6) and (7).

The values of 'variability of system' and 'coefficient of variation'for various values of $k, \in$ forfixed values of $\lambda, \mu$ are computed which are given in tables-5.7\& 5.9. The values of 'variability of the system' and 'coefficient of variation' for fixed values of $k, \in$ and for various values of $\lambda, \mu$ are provided in tables (5.8) and (5.10).

From equation-9 a\& from the corresponding table we can observe that as $\mu$ increases the "variability of the system size" decreases and "coefficient of variation" increases. As $\lambda$ increases and for fixed values of $\mu, \in$ and $k$, the "variability of the system size' increases $\&$ the 'coefficient of variation decreases. We may observe that as $\in$ increases the 'variability of the system size, decreases and 'coefficient of variation' increases for fixed values of $\lambda, \mu$ and $k$. As $k$ increases, the 'variability of the system' decreases and the 'coefficient of variation increases'.

For this model $\in=0$ and $k=1$ reduces to $M / M / 1$ classical model. The mean-queue length $\&$ 'variability of the system size' of this model are less than that of the classical. When $k=1$, this model becomes $M / M /$ 1independent model for $\epsilon=0$, this model is same as $M / M^{[K]} / 1$ model.

TABLE 1.1

VALUES OF $P_{0}$

$\lambda=3, \mu=5$

\begin{tabular}{|c|c|c|c|c|c|}
\hline \multicolumn{7}{|c|}{$\boldsymbol{\lambda = \mathbf { 3 }}, \boldsymbol{\mu}=\mathbf{5}$} & $\mathbf{0 . 6}$ & $\mathbf{0 . 8}$ \\
\hline $\boldsymbol{K} / \epsilon$ & $\mathbf{0 . 0}$ & $\mathbf{0 . 2}$ & $\mathbf{0 . 4}$ & 0.4545 & 0.4762 \\
\hline $\mathbf{1 .}$ & 0.4000 & 0.4167 & 0.4348 & 0.6081 & 0.6203 \\
\hline $\mathbf{2 .}$ & 0.5780 & 0.5871 & 0.5971 & 0.6357 & 0.6459 \\
\hline $\mathbf{3 .}$ & 0.6106 & 0.6182 & 0.6264 & 0.6433 & 0.6529 \\
\hline $\mathbf{4 .}$ & 0.6201 & 0.6270 & 0.6347 & 0.6458 & 0.6551 \\
\hline $\mathbf{5 .}$ & 0.6214 & 0.6300 & 0.6374 & & \\
\hline
\end{tabular}

(TABLE 1.2)

"VALUES OF $P_{0}$ "

for $K=2 \& \in=5$

\begin{tabular}{|c|c|c|c|c|c|}
\hline $\boldsymbol{\mu} / \boldsymbol{\lambda}$ & $\mathbf{1}$ & $\mathbf{2}$ & $\mathbf{3}$ & $\mathbf{4}$ & $\mathbf{5}$ \\
\hline $\mathbf{1 .}$ & 0.9024 & 0.7681 & 0.6548 & 0.5551 & 0.4649 \\
\hline $\mathbf{2 .}$ & 0.9161 & 0.7983 & 0.6975 & 0.6081 & 0.5269 \\
\hline $\mathbf{3 .}$ & 0.9265 & 0.8214 & 0.7305 & 0.6493 & 0.5752 \\
\hline $\mathbf{4 .}$ & 0.9345 & 0.8397 & 0.7568 & 0.6823 & 0.6141 \\
\hline $\mathbf{5 .}$ & 0.9410 & 0.8545 & 0.7783 & 0.7094 & 0.6461 \\
\hline
\end{tabular}


TABLE 1.3

VALUES OF $L$

$\lambda=3 \quad, \mu=5$

\begin{tabular}{|c|c|c|c|c|c|}
\hline $\boldsymbol{K} / \boldsymbol{E}$ & $\mathbf{0 . 0}$ & $\mathbf{0 . 2}$ & $\mathbf{0 . 4}$ & $\mathbf{0 . 6}$ & $\mathbf{0 . 8}$ \\
\hline $\mathbf{1 .}$ & 1.5000 & 1.3998 & 1.2999 & 1.2002 & 1.1000 \\
\hline $\mathbf{2 .}$ & 0.7301 & 0.7033 & 0.6748 & 0.6445 & 0.6121 \\
\hline $\mathbf{3 .}$ & 0.6377 & 0.6176 & 0.5964 & 0.5731 & 0.5482 \\
\hline $\mathbf{4 .}$ & 0.6126 & 0.5949 & 0.5755 & 0.5545 & 0.5316 \\
\hline $\mathbf{5 .}$ & 0.6093 & 0.5873 & 0.5689 & 0.5485 & 0.5295 \\
\hline
\end{tabular}

TABLE 1.4

VALUES OF $L$

\begin{tabular}{|c|c|c|c|c|c|}
\hline \multicolumn{7}{|c}{$\boldsymbol{K}=\mathbf{2}$} & $\boldsymbol{\epsilon}=\mathbf{0 . 4}$ & $\mathbf{4}$ & $\mathbf{5}$ \\
\hline $\boldsymbol{\mu} / \boldsymbol{\lambda}$ & $\mathbf{1}$ & $\mathbf{2}$ & $\mathbf{3}$ & 0.8015 & 0.1510 \\
\hline $\mathbf{1 .}$ & 0.1082 & 0.3019 & 0.5272 & 0.6445 & 0.8979 \\
\hline $\mathbf{2 .}$ & 0.0916 & 0.2527 & 0.4337 & 0.5401 & 0.7385 \\
\hline $\mathbf{3 .}$ & 0.0793 & 0.2174 & 0.3689 & 0.4656 & 0.6284 \\
\hline $\mathbf{4 .}$ & 0.0701 & 0.1909 & 0.3214 & 0.4096 & 0.5477 \\
\hline $\mathbf{5 .}$ & 0.0627 & 0.1703 & 0.2849 & & \\
\hline
\end{tabular}

TABLE 1.5

VALUES OF $L_{q}$

$\lambda=3 \quad, \quad \mu=5$

\begin{tabular}{|c|c|c|c|c|c|}
\hline $\boldsymbol{K} / \epsilon$ & $\mathbf{0 . 0}$ & $\mathbf{0 . 2}$ & $\boldsymbol{0 . 4}$ & $\mathbf{0 . 6}$ & $\mathbf{0 . 8}$ \\
\hline 1. & 0.9000 & 0.8165 & 0.7347 & 0.6547 & 0.5762 \\
\hline $\mathbf{2 .}$ & 0.3081 & 0.2904 & 0.2228 & 0.2526 & 0.2324 \\
\hline $\mathbf{3 .}$ & 0.2483 & 0.2219 & 0.2228 & 0.2088 & 0.1941 \\
\hline 4. & 0.2327 & 0.2219 & 0.2102 & 0.1978 & 0.1845 \\
\hline $\mathbf{5 .}$ & 0.2307 & 0.2173 & 0.2063 & 0.1943 & 0.1816 \\
\hline
\end{tabular}

TABLE 1.6

VALUES OF $L_{q}$

$K=\mathbf{2}, \quad \in=\mathbf{0 . 4}$

\begin{tabular}{|c|c|c|c|c|c|}
\hline $\boldsymbol{\mu} / \boldsymbol{\lambda}$ & $\mathbf{1}$ & $\mathbf{2}$ & $\mathbf{3}$ & $\mathbf{4}$ & $\mathbf{5}$ \\
\hline $\mathbf{1 .}$ & & 0.700 & 0.1820 & 0.3566 & 0.6159 \\
\hline $\mathbf{2 .}$ & 0.0077 & 0.510 & 0.1321 & 0.2526 & 0.4248 \\
\hline $\mathbf{3 .}$ & 0.0058 & 0.0388 & 0.0994 & 0.1894 & 0.3137 \\
\hline $\mathbf{4 .}$ & 0.0046 & 0.0306 & 0.0782 & 0.1479 & 0.2425 \\
\hline $\mathbf{5 .}$ & 0.0037 & 0.0248 & 0.632 & 0.1190 & 0.1938 \\
\hline
\end{tabular}

\section{REFERENCE}

1. Baccelli, F. Mssey, W. A. (1989): “A Sample Path Analysis Of The M/M/1 Queue”, J Appl. Prob. 26, 418-422.

2. Boxma, O.J., Kelly, F.P. And Konheim, A. G. (1984): "The Product Form For Sojourn Time Distributions Incyclic Exponential Queues”,J. Assoc. Comput. Mach., 31, 128-133.

3. Brien O . (1987) : "Extreme Values For Stationary And Markov Sequences" Journal. Soci. Indian Appl. Math ., 2, 133.

4. Chae, K. C ., Lee, H. W. And Ahn C. W. (2001): “An Arrival Time Approach To M/G/1 Type Queues With Generalized Vacations", Queueing Systems 38, 91- 100.

5. Chaudhary , G . (1998) : "On A Batch Arrival Poisson Queue With A Random Setup And Vacation Period”, Comm .Ops. Res., 25 , 1013-1026.

6. Chhikra (1972) : Statistical Inference Related To The Inversegaussian Distribution, Ph.D. Dissertation, Oklahoma State University .

7. Conti, P.L. And Giovanni , L.D. (2002) : “Queueing Models And Statistical Analysis For Atm Based Networks" ,Sankhaya : The Indian Journal Of Statistics , 64, 50-75. 
8. Cooper, R.B.(1970) : “Queues Served In Cyclic Order : Waiting Times”, Bell System Technical Journal , 49 399- 413 .

9. Erlang, A.K. (1909) : "Probability And Phone Calls" ,Nyt. Tidsskr Mat. Ser. B., 20 , 33-39.

10. Fry , T.C. (1982) : "The Theory Of Probability As Applied To Problems Of Congestion In Probability And Its Engineering Uses", D.Van Nostrand Co . Inc., Princeton New Jersy.

11. Griffitins , J.D ., Leomenko, G.M., Williams , J .E. (2006) : The Transient Solution To M/E/1 Queue , Oper. Res. Letters, Vol. 34 , Issue 4 Pp. 349-354.

12. Hiller, F.S. And Boiling, R.W. (1967) : Finite Queues In Series With Exponential Or Erlang Service Time-A Numerical Approach”, Operation Research ., 15 , 286-303.

13. Singla, Neelam. "Busy Period Analysis Of A Markovian Feedback Queueing Model With Servers Having Unequal Service Rate." International Journal Of Applied Mathematics \& Statistical Sciences (Ijamss) 6.1 (2017) 55-64

14. Ghimire, Sushil, Et Al. "Multi-Server Batch Service Queuing Model With Variable Service Rates." International Journal Of Applied Mathematics \& Statistical Science (Ijamss) 6.4 (2017): 43-54.

15. Mohamad, H. A., And Sattar Naser Ketab. "Oscillation And Nonoscillation Properties Of Solutions Of Third Order Linear Neutral Differential Equations." International Journal Of Applied Mathematics \& Statistical Sciences (Ijamss) 5.3 (2016): 31-38.

16. Shaalini, J. Vinci, And A. Emimal Kanaga Pushpam. "Application Of Rkhem Method For Solving Delay Differential Equations With Constant Lags." International Journal Of Applied Mathematics \& Statistical Sciences (Ijamss) 5.3 (2016) 39-46

17. Muxamediyeva, D. K. "Properties Of Self Similar Solutions Of Reaction-Diffusion Systems Of Quasilinear Equations." International Journal Of Mechanical And Production Engineering Research And Development (Ijmperd) 8.2 (2018) 555-566

18. Jawahar, G. Gomathi. "Qualitative Analysis On Second Order Neutral Delay Difference Equations." International Journal Of Mechanical And Production Engineering Research And Development (Ijmperd) 9.2 (2019) 659-664 